\title{
Antigenic and Structural Relationships of the Surface Antigens of Hepatitis B Virus, Ground Squirrel Hepatitis Virus, and Woodchuck Hepatitis Virus
}

\author{
MARK A. FEITELSON, PATRICIA L. MARION, AND WILLIAM S. ROBINSON* \\ Department of Medicine, Stanford University School of Medicine, Stanford, California 94305
}

Received 3 February 1981/Accepted 15 April 1981

The surface antigens of human hepatitis B (HBsAg), ground squirrel hepatitis (GSHsAg), and woodchuck hepatitis (WHsAg) viruses were compared serologically, and their major polypeptides were analyzed by sodium dodecyl sulfatepolyacrylamide gel electrophoresis and tryptic peptide mapping. Results showed that both GSHsAg and WHsAg are antigenically cross-reactive, that their major pairs of polypeptides have identical mobilities on sodium dodecyl sulfate gels, and that the major polypeptides of GSHsAg and WHsAg migrate faster in sodium dodecyl sulfate-polyacrylamide gel electrophoresis than do the corresponding bands of HBsAg. The peptide maps of the major (P-22) surface antigen polypeptides of GSHsAg and WHsAg showed that they shared over half of their spots. Peptide mapping of HBsAg subtypes indicated a close relationship between the major polypeptides (P-24) of $a d w$ and $a d r$ and a more distal relationship to ayw. Only about $25 \%$ of the spots shared by the combined HBsAg subtypes were also found in the peptide maps of GSHsAg and WHsAg, indicating at least some structural homology among the major polypeptides of the human and animal virus surface antigen particles. This is also reflected in the serological crossreactivity among HBsAg, GSHsAg, and WHsAg. Further, the detection of ground squirrel and woodchuck antigens by Ausria II radioimmunoassay, combined with peptide mapping data indicating the common origin of these viruses, suggests that the common $a$ determinant is shared by each and is restricted to approximately $25 \%$ of the sequences in their major polypeptides.

The recent discoveries of hepatitis B-like viruses in ground squirrels (13) and woodchucks (20) provide important animal models for the study of viral infections and disease produced by viruses of this class. Hepatitis B virus (HBV)-, ground squirrel hepatitis virus (GSHV)-, and woodchuck hepatitis virus (WHV)-associated structures in serum of infected individuals include 42- to 45-nm-diameter virions and smaller (approximately 16- to 25-nm-diameter) spherical and filamentous particles. The latter two particles, as well as virions, bear the viral surface antigen which is contained in virus-specific polypeptides (reviewed in reference 16). The small spherical and filamentous particles, however, lack detectable viral core antigen, viral DNA, and the other activities associated with the virion core. These particles are usually present in serum in much higher concentrations than are virions.

The surface antigen particles of HBV, GSHV, and WHV (bearing the antigens designated HBsAg, GSHsAg, and WHsAg, respectively) share similar morphology and buoyant density, and all induce antibodies which precipitate the homologous antigens $(8,13,20,21)$. GSHsAg and WHsAg, however, demonstrate only low levels of cross-reactivity with $\mathrm{HBsAg}$ (8, 21), whereas serological relationships between WHsAg and GSHsAg have not yet been ascertained. It is well known that HBsAg is antigenically complex, with an $a$ or group-specific determinant present in all HBsAg particles and usually a pair of type-specific determinants ( $d$ or $y$ and $w$ or $r$, each characteristic of some but not all HBsAg preparations $(1,12)$. These define the HBV genotype. Recently, some antigenic heterogeneity has been detected in GSHsAg with antibodies directed against the common $a$ determinant of HBsAg (8), although the relationship between this heterogeneity and that in HBsAg remains to be clarified.

WHV and GSHV DNAs have recently been shown to share homology with HBV DNA (6, $19,21)$. The extent of homology, at least between the woodchuck and human viruses, is very low 
(21), and preliminary restriction endonuclease analyses of WHV (6) and GSHV (19) DNAs also suggest little similarity to HBV DNA. However, the restriction site differences do not necessarily indicate the degree of genetic relatedness of these viruses. The coding sequences for the surface antigen polypeptides in HBV DNA hybridize with GSHV DNA (19).

To further develop these animal viruses as useful models for HBV in humans, a more refined knowledge of the molecular relationships among them is desirable. In this report the polypeptides of the small spherical HBsAg (subtypes $a d w, a y w$, and $a d r)$, GSHsAg, and WHsAg particles are compared serologically, by sodium dodecyl sulfate-polyacrylamide gel electrophoresis (SDS-PAGE), and by tryptic peptide mapping.

\section{MATERIALS AND METHODS}

Isolation of HBsAg, GSHsAg, and WHsAg. Discarded HBsAg-positive blood units high in HBV DNA polymerase activity $(10,13)$ and in surface antigen titer by radioimmunoassay (Ausria II, Abbott Laboratories) were subtyped with appropriate antisera by immunodiffusion (5). Five blood bank units of determined subtype were chosen for analysis (adw 1447, $a d w 1419$, ayw 1392, ayw 1364, and adr 1460). Two ground squirrel (GL20 and PC43) and two woodchuck (WHV-1 and WHV-3 [supplied by L. I. Lutwick]) sera which were positive by Ausria II and high in virion DNA polymerase activity were used as starting materials for GSHsAg and WHsAg isolation, respectively. Due to the relatively large volumes and low concentrations of $\mathrm{HBsAg}$ compared with GSHsAg and WHsAg, the HBsAg from blood bank units were partially purified and concentrated by polyethylene glycol precipitation (8). Samples of animal sera (2 to $3 \mathrm{ml}$ ) and redissolved polyethylene glycol precipitates of human sera were each chromatographed over a BioGel A-5M column, concentrated, and centrifuged to equilibrium in a discontinuous $\mathrm{CsCl}$ density gradient as previously described (8). After each step, HBsAg was detected by electroimmunodiffusion $(8,9)$; GSHsAg was detected by electroimmunodiffusion, using a monoclonal antibody to GSHsAg; and WHsAg was detected by Ausria II. Purified preparations of the surface antigens produced a single, symmetrical peak with a low, 280-nm absorbance background when recentrifuged on $\mathrm{CsCl}$ density gradients. The peak of antigenic activity in each case corresponded to the single peak of 280-nm absorbance at a solution density of approximately $1.2 \mathrm{~g} / \mathrm{ml}$. UV absorption profiles of the surface antigen preparations indicated peaks at 280 and $290.5 \mathrm{~nm}$, the latter being characteristic of the high tryptophan/tryosine ratio present in surface antigen and seen only in highly purified preparations.

Serological assays. To ascertain the extent of serological cross-reactivity among $\mathrm{HBsAg}$, GSHsAg, and WHsAg, radioimmunoprecipitation was used. Anti-HBs/ad raised in guinea pigs was a gift of J. L. Gerin. Anti-GSHs was produced in mice by immunization with the appropriate antigen, followed by de- termination of antibody titers with the homologous antigen. Anti-WHs was found in two woodchuck sera which were negative in both DNA polymerase and Ausria II assays. For precipitation, $60,000 \mathrm{cpm}$ of ${ }^{125} \mathrm{I}$ labeled antigen, labeled after purification by the iodogen procedure $(7,8)$, was added to 10 -fold dilutions of antibody in phosphate-buffered saline containing $0.1 \%$ bovine serum albumin in a total volume of $25 \mu$ l. The mixture was incubated at $37^{\circ} \mathrm{C}$ for $1 \mathrm{~h}$ and then overnight at $4^{\circ} \mathrm{C}$. A $25-\mu l$ portion of Sepharose CL-4Blinked protein A beads $(0.11 \mathrm{~g} / \mathrm{ml}$; Pharmacia Fine Chemicals, Inc.) was added to each mixture, and the mixtures were incubated for $45 \mathrm{~min}$ at room temperature. The Sepharose beads were then trapped on glass fiber filters, washed thoroughly with phosphatebuffered saline containing $0.1 \%$ bovine serum albumin, and counted in a gamma counter.

Radiolabeling and chemical modification of HBsAg, GSHsAg, and WHsAg. Highly purified preparations of surface antigen particles were dialyzed against $0.01 \mathrm{M}$ sodium borate buffer $(\mathrm{pH} 8.5)-0.1 \%$ SDS, lyophilized, and labeled with Bolton-Hunter reagent (2) as previously described (8). After radioacylation, the reaction was driven to completion with an excess of unlabeled ester (TAGIT, Calbiochem). Reductive methylation was carried out as previously described (14), with minor modifications (Feitelson et al., submitted for publication) to ensure that all lysyl residues were resistant to trypsinization, which followed separation of the polypeptides by SDS-PAGE. Polypeptides were then reduced with 2-mercaptoethanol, alkylated with iodoacetamide, and desalted over a column of Bio-Gel P-10 (8).

As mentioned in our previous study (8), radioacylation of amino acids and small peptides with the Bolton-Hunter reagent introduces chemical heterogeneity, possibly due to several factors: the short halflife of the Bolton-Hunter reagent in aqueous media, the different intrinsic reactivities of various primary amino groups, the bulk of the amino acid residues immediately surrounding the reactive primary amino group, and the Bolton-Hunter reagent/protein ratio, as well as other factors. This heterogeneity is limited by addition of a vast excess of cold reagent after radioacylation. Although the number of spots in the final peptide maps is greater than would be predicted from the primary structure, the percent homology values reported are very similar to those achieved by direct iodination (chloramine- $\mathrm{T}$ ) procedures (unpublished data). Experience has shown that, in comparing the peptide maps of two or more proteins labeled by the Bolton-Hunter reagent, the chemical heterogeneity introduced does not alter the percent homology obtained by conventional methods.

SDS-PAGE and peptide mapping. Discontinuous SDS-PAGE was carried out as described by Laemmli (11) with several modifications (8; Feitelson et al., submitted for publication). Fixed and dried gels were exposed for autoradiography, and individual bands were removed and trypsinized. Peptide maps of each digest were generated on thin-layer cellulose plates (Brinkmann Instruments Inc.) by electrophoresis in the first dimension and ascending chromatography in the second dimension and were detected by autoradiography (8). 


\section{RESULTS}

Serological relationship among HBsAg, GSHsAg, and WHsAg. Radiolabeled HBsAg, GSHsAg, and WHsAg preparations were mixed with different dilutions of antisera raised to the homologous and heterologous antigens and precipitated with protein A immobilized on Sepharose CL-4B beads. The results (Fig. 1), demonstrate serological cross-reactivity among the three types of surface antigen particles. The abilities of anti-GSHs to bind WHsAg (Fig. 1A) and anti-WHs to precipitate GSHsAg (Fig. 1B) indicate serological cross-reactivity of the surface antigens of these viruses. GSHsAg and WHsAg are probably not identical since the curves with homologous and heterologous systems are different. Further, WHsAg could not be precipitated with a potent monoclonal antiGSHs, indicating the complete absence of a determinant on WHsAg, which was characteristic of GSHsAg. The detection of both GSHsAg and WHsAg with anti-HBs in the above assay (Fig. $1 \mathrm{~A}$ and $\mathrm{B}$ ), as well as by Ausria II, indicates that determinants on $\mathrm{HBsAg}$ are also present on the antigens of the other two viruses. The precipitation of HBsAg with anti-WHs (Fig. 1C) in comparison to that with homologous antisera confirms the partial cross-reactivity noted above, whereas the failure of an anti-GSHs-containing serum to precipitate $\mathrm{HBsAg}$ indicates that the antiserum contained no antibodies which would react with $\mathrm{HBsAg}$.

Relationship between the major polypeptides of GSHsAg and WHsAg. SDS-PAGE of radioacylated GSHsAg (from GL20) and WHsAg (from WHV W $_{1}$ revealed a pair of major bands at 22,000 (P-22) and 25,000 (P-25) daltons (Fig. 2). Similar analysis of GSHsAg from another ground squirrel (PC43) and WHsAg from another woodchuck $\left(\mathrm{WHV}_{3}\right)$ produced identical results. The comigration of the GSHV and WHV polypeptides and the faster migration of the GSHV polypeptides in comparison to the analogous pair in HBsAg (8) indicate that the WHsAg major components are similarly different from the major polypeptides of HBsAg. Indeed, SDS-PAGE analysis of WHsAg and

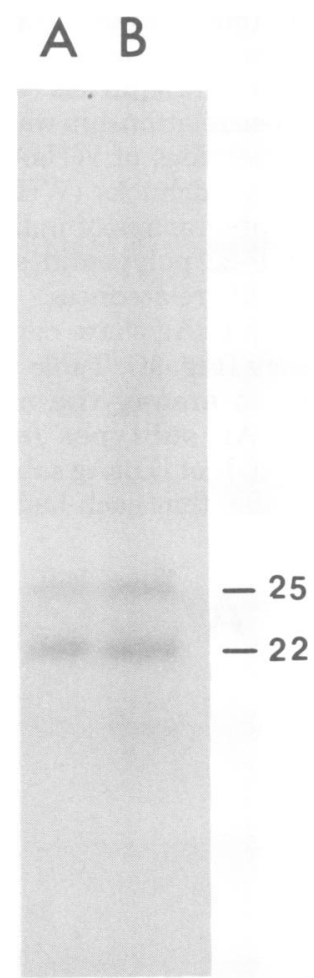

Fig. 2. SDS-PAGE of (A) GSHsAg and (B) WHsAg. Purified surface antigen particles were disrupted and labeled by the Bolton-Hunter reagent procedure as described in the text. After electrophoresis, the dried gel was exposed for $30 \mathrm{~min}$ by autoradiography.

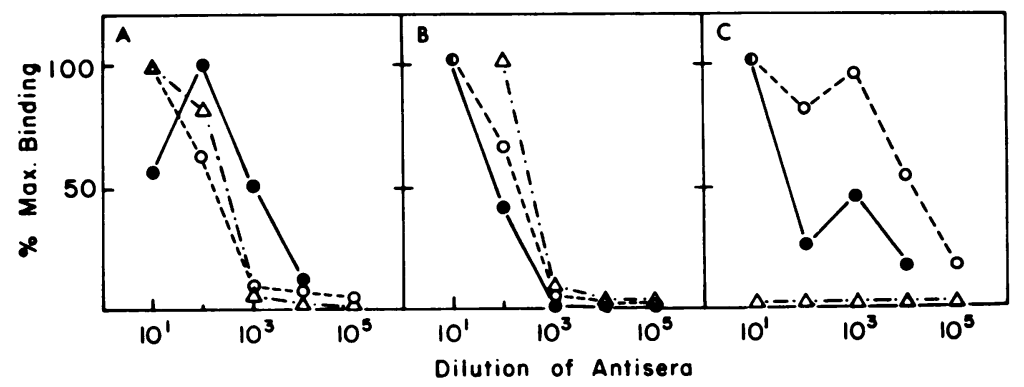

Fig. 1. Serological relationships among $\mathrm{HBsAg}, \mathrm{GSHsAg}$, and WHsAg. Purified surface antigens from each source were labeled by the iodogen procedure, mixed with increasing dilutions of antibody directed against the homologous or heterologous antigens, and finally precipitated with immobilized protein $A$ (see text). The antisera used were: anti-WHs (O); anti-GSHs $(\triangle)$; anti-HBs $(O)$. (A) ${ }^{125} I$-labeled WHsAg; (B) ${ }^{125 I-}$ labeled GSHsAg; (C) ${ }^{125}$ I-labeled HBsAg. 
HBsAg polypeptides demonstrated this difference (data not shown).

Peptide mapping of the $\mathrm{P}-22$ polypeptides of GSHsAg (GL20 and P43) and WHsAg (WHV and $\mathrm{WHV}_{3}$ ) was carried out (Fig. $3 \mathrm{~A}$ and $\mathrm{B}$ ), and the relationships among different polypeptide pairs are summarized in Table 1. A high degree of relatedness (about 75\%) was found within the maps of P-22 isolated from GSHsAg particles of two different ground squirrels (GL20 and PC43). A similarly close relationship was found between the $\mathrm{P}-22$ polypeptides of $\mathrm{WHsAg}$ isolated from two different woodchucks $\left(\mathrm{WHV}_{1}\right.$ and $\left.\mathrm{WHV}_{3}\right)$. Composite peptide maps of individual GSHsAg and WHsAg P-22 polypeptides also showed a high degree of relatedness, indicating that GSHsAg and WHsAg share considerable structural homology (Fig. 3C; Table 1).

Relationship among the major polypeptides of HBsAg subtypes (adw, ayw, and $a d r)$. SDS-PAGE of HBsAg subtypes $a d w$, ayw, and $a d r$ revealed that each had a pair of major
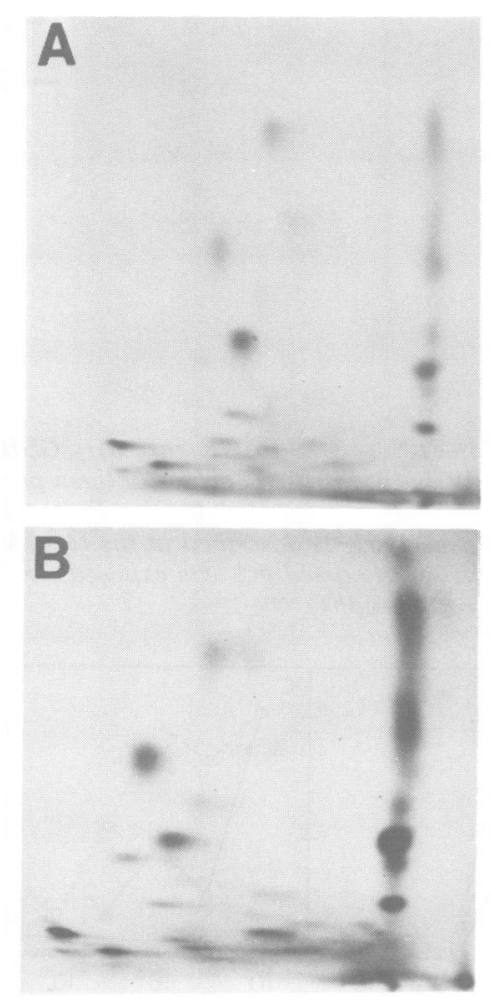

polypeptides with apparent molecular weights of 24,000 (P-24) and 28,000 (P-28) (data not shown), as previously described (18). Peptide maps of the P-24 of each subtype are shown in Fig. 4A to C, and the homology among individual composites is summarized in Table 2 . Within the $a d w$ or ayw subtype, composites of P-24 from different patients showed a high extent of homology, similar to that observed for GSHsAg and WHsAg (Table 1). The composite of adw and $a d r$ suggests that these two subtypes are very closely related. Comparison of the peptide maps of ayw (from either patient) with those of $a d w$ or $a d r$, however, suggests that the P-24 polypeptide of ayw is not as closely related to $a d w$ or $a d r$ as are the latter two to each other.

Homology among HBsAg subtypes, GSHsAg, and WHsAg. The close relationships seen in the peptide map composites of different $a d w$ samples (1447 and 1419), of different ayw samples (1392 and 1364), and of $a d w$ plus $a d r$ are in contrast to the substantially lower per-

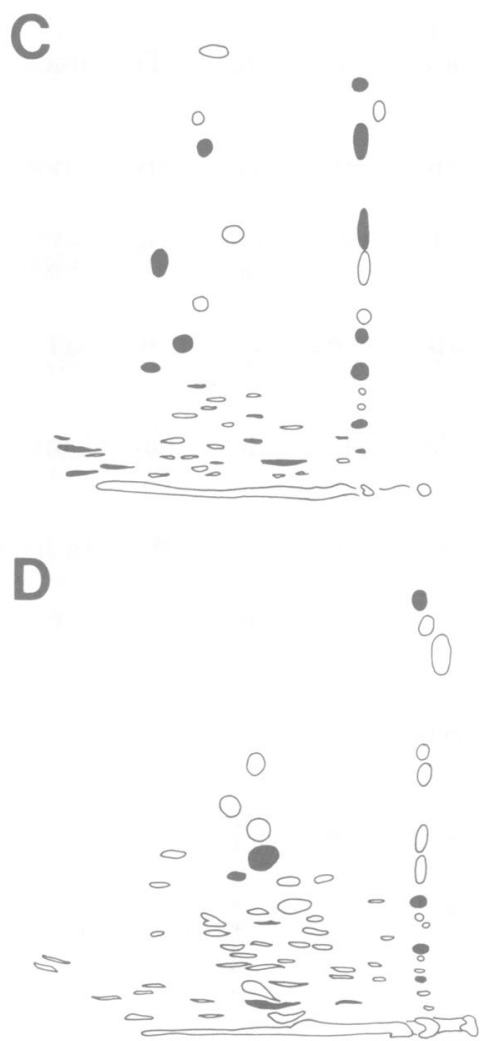

Fig. 3. Peptide maps of P-22 of GSHsAg and WHsAg. (A) GSHsAg (GL20); (B) WHsAg (WHV $)$; (C) composite of GSHsAg (GL20, PC43) and WHsAg (WHV,$\left.W H V_{3}\right)$ peptide maps on the background of P.22 of GL20; (D) composite of GSHsAg and WHsAg [composite in (C)] with HBsAg (adw + ayw + adr, Fig. 4D) polypeptides on the background of P-24 of ayw 1392. Blackened spots are those which are common to all of the polypeptides making up the composite. 
centage of spots common to all samples of each subtype (Fig. 4D; Table 3 ). An average of $30 \%$ of the spots in the peptide maps of ayw samples

TABLE 1. Relationship between the 22,000-dalton polypeptides of GSHsAg and WHsAg

\begin{tabular}{lcrc}
\hline \multirow{2}{*}{ Polypeptide } & \multicolumn{3}{c}{ Homology (\%) $^{a}$} \\
\cline { 2 - 4 } & GL20 & PC43 & WHV $_{3}$ \\
\hline GL20 & 100 & 75 & 73 \\
PC43 & & 100 & 85 \\
WHV $_{1}$ & 79 & 69 & 76 \\
\hline
\end{tabular}

${ }^{a}$ Extent of structural homology is expressed as a percentage of spots on a given peptide map in common with those in the peptide map of another protein. The values reported are averages because not all of the comparisons made are between proteins having the same number of spots. Each percentage is based upon at least two separate peptide maps of each digest. The small variations in the number of spots noted from plate to plate may be due to differences in electrophoresis or chromatography or to some spontaneous deamination or oxidation or both of some amino acid residues. For all polypeptides analyzed herein, the average amount of variability due to these factors is approximately $8 \%$ of the spots on each peptide map. were also found in the maps of the $a d w$ and $a d r$ major 24,000 -dalton polypeptides. Nearly $60 \%$ of the spots in the $a d w$ and $a d r$ peptide maps, however, were identical to spots in the ayw maps. The composite in Fig. 4D shows these peptides which are conserved among the three subtypes. Since the different $\mathrm{HBsAg}$ subtypes share a common antigenic determinant, denoted as $a$, it is likely that this determinant is included among these conserved peptides.

The homology noted above in individual composites of GSHsAg and WHsAg polypeptides (Table 1) included subsets of spots which were not common to all samples of both GSHsAg and WHsAg 22,000-dalton polypeptides. The composite shown in Fig. 3C (GL20, PC43, $\mathrm{WHV}_{1}$, and $\mathrm{WHV}_{3}$ ) demonstrates this and shows, together with Table 3 , that the number of spots shared among multiple samples of WHsAg and GSHsAg was approximately $55 \%$. Analysis of the 22,000-dalton polypeptide from a third woodchuck and ground squirrel did not change the overall percent homology shown in Fig. $3 \mathrm{C}$ and reported in Table 3. Clearly, then, at least a simple majority of the peptides in GSHsAg and
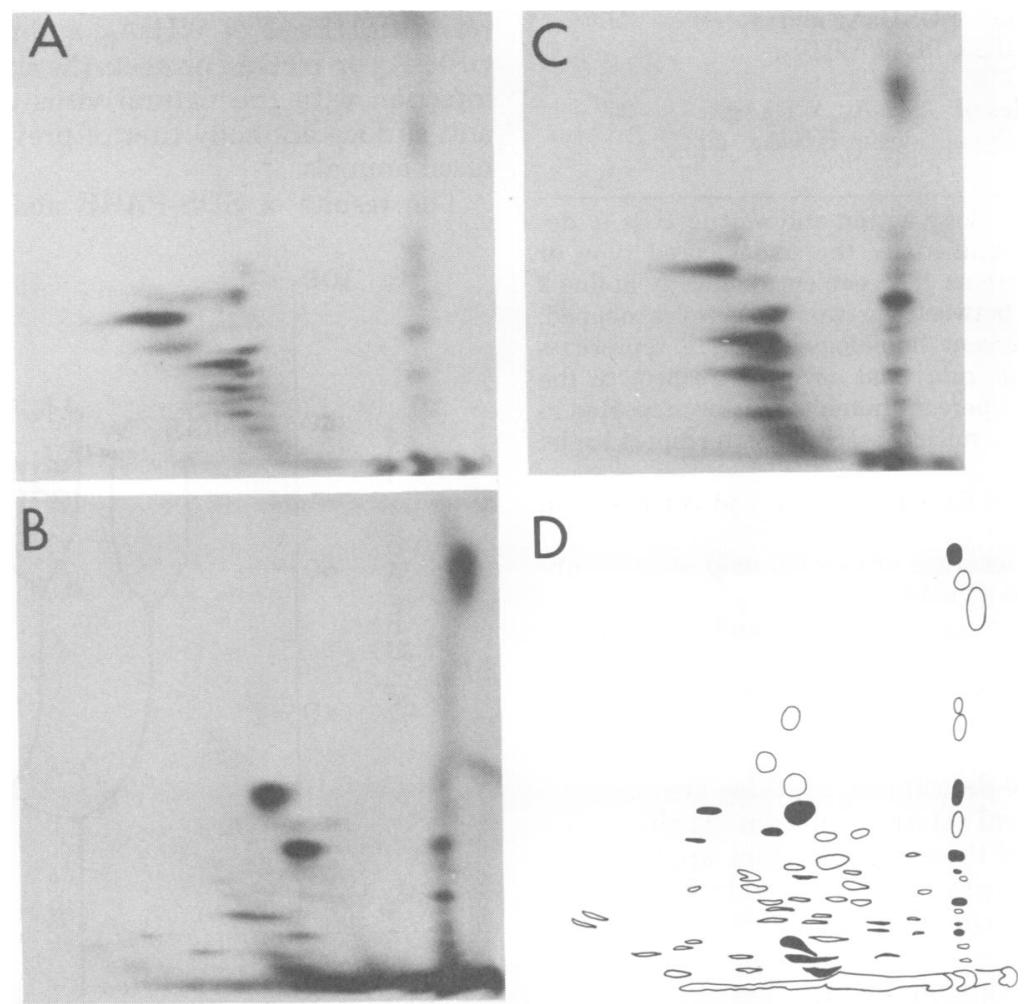

Fig. 4. Peptide maps of P-24 from various HBsAg subtypes. (A) adw 1447; (B) ayw 1392; (C) adr 1460; (D) composite of adw 1447 and adw 1419 and ayw 1392, 1364, and adr 1460 P-24 polypeptides on the background of ayw 1392. Blackened spots are shared by all of the polypeptides in this composite. 
TABLE 2. Relationship between the 24,000-dalton polypeptides of HBsAg subtypes

\begin{tabular}{crrcc}
\hline & \multicolumn{4}{c}{ Homology (\%) } \\
\cline { 2 - 5 } Polypeptide & ayw & $a d w$ & $a d w$ & $a d r$ \\
& 1364 & 1447 & 1419 & 1460 \\
\hline ayw 1364 & 100 & 65 & 68 & 61 \\
ayw 1392 & 83 & 59 & 70 & 60 \\
adw 1447 & & 100 & 76 & 87 \\
\hline
\end{tabular}

${ }^{a}$ See footnote $a$, Table 1 .

TABLE 3. Relationship among major surface antigen polypeptides of $\mathrm{HBsAg}, \mathrm{GSHsAg}$, and WHsAg by tryptic peptide mapping

\begin{tabular}{|c|c|}
\hline Composite $^{a}$ & $\begin{array}{l}\text { Homol- } \\
\text { ogy }(\%)\end{array}$ \\
\hline $\begin{array}{l}\text { adw 1447, adw 1419, adr } 1460 \\
\text { ayw 1364, ayw } 1392\end{array}$ & $\begin{array}{l}59^{b} \\
83^{b}\end{array}$ \\
\hline $\begin{array}{l}\text { All subtypes } \\
\text { ayw }(1364,1392) \\
\text { adw }(1447,1419) \\
\text { adr }(1460)\end{array}$ & $\begin{array}{l}30^{b, c} \\
61^{b, c} \\
56^{b, c}\end{array}$ \\
\hline $\begin{array}{l}22 \mathrm{~K} \text { polypeptides of GSHsAg (GL20, } \\
\text { PC43) }\end{array}$ & $75^{d}$ \\
\hline $\begin{array}{l}22 \mathrm{~K} \text { polypeptides of WHsAg }\left(\mathrm{WHV}_{1} \text {, }\right. \\
\left.\mathrm{WHV}_{3}\right)\end{array}$ & $76^{d}$ \\
\hline $\begin{array}{l}22 \mathrm{~K} \text { polypeptides of GSHsAg and } \\
\mathrm{WHsAg}_{\left(\mathrm{GL} 20, \mathrm{PC}, \mathrm{WHV}_{1}\right.} \\
\left.\mathrm{WHV}_{3}\right)\end{array}$ & $55^{d}$ \\
\hline $\begin{array}{l}22 \mathrm{~K} \text { polypeptides of GSHsAg, WHsAg, } \\
\text { and } 24 \mathrm{~K} \text { polypeptides of } \mathrm{HBsAg} \text { (all } \\
\text { subtypes) }\end{array}$ & $25^{e}$ \\
\hline
\end{tabular}

${ }^{a}$ Percent homology within and among HBsAg determinants is signified by the underscored type or subtype designations. The percent homology in line 2 represents that between the two ayw samples mapped, whereas the percent homology in line 3 represents that among $a y w, a d w$, and $a d r$ with respect to the map of $a y w$. The percent homology values reported in lines 4 and 5 are similarly assigned with respect to the subtype listed.

${ }^{b}$ Derived from data in Table 2 and composite in Fig. 4.

${ }^{c}$ Average percentage of peptide map of each subtype common to all subtypes.

${ }^{d}$ Derived from data in Table 1 and composite in Fig. 3.

${ }^{e}$ Derived from data in Tables 1 and 2 and composite in Fig. 3.

WHsAg 22,000-dalton polypeptides are shared.

The structural relationships among the major polypeptides of these virus surface antigen particles can be diagramed, as shown in Fig. 5. This figure implies that HBV, GSHV, and WHV share a common origin and may have arisen during evolution from a single hepatitis-like virus. The divergence of woodchuck and ground squirrel viruses, as well as the appearance of $d$ and $y$ determinants of HBsAg, appear to have occurred earlier than the divergence of the $r$ determinant of $\mathrm{HBsAg}$ and the heterogeneity within single $\mathrm{HBsAg}$ subtypes, GSHsAg, and WHsAg from different sources.

\section{DISCUSSION}

The relationships among intact $\mathrm{HBsAg}$, GSHsAg, and WHsAg particles were studied by radioimmunoprecipitation, and the relationships among the major nonglycosylated polypeptides of each were analyzed by SDS-PAGE and tryptic peptide mapping. The results of radioimmunoprecipitation demonstrated the presence of one or more antigenic determinants conserved among the three surface antigen particles (Fig. 1). Further, the results suggest that there is only partial cross-reactivity among the antigens and imply the presence of unique determinants in the antigen of each virus. The detection of the HBsAg common determinant $a$ by Ausria II, combined with the detection of GSHsAg and WHsAg by this assay, suggests that the portion of each antigen which is conserved may be all or part of this determinant. It will be interesting to determine whether immunization of woodchucks with HBsAg or GSHsAg, ground squirrels with $\mathrm{HBsAg}$ or WHsAg, and primates with GSHsAg or WHsAg protects the animals against infection with the natural virus or boosts the anti-surface antibody titer of previously immunized animals.

The results of SDS-PAGE analysis indicate

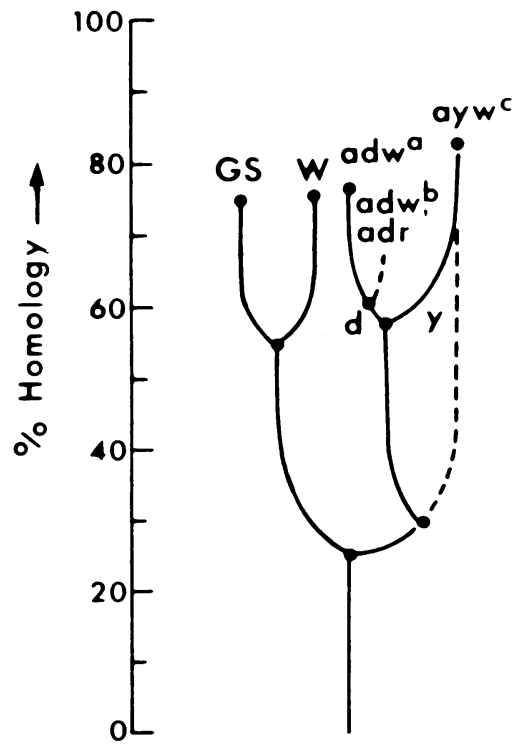

FIg. 5. Structural relationships among $\mathrm{GSHs} A g$, WHsAg, and HBsAg (adw, ayw, and adr) based upon homology indicated by their peptide maps. 
that the major polypeptide pairs of GSHsAg and WHsAg comigrate (Fig. 2) and move faster on the gel than does the analogous pair of HBsAg polypeptides (data not shown), suggesting greater similarity between GSHsAg and WHsAg than between either of the latter and HBsAg. The conclusion is strengthened by the peptide mapping data summarized in Table 3 and Fig. 5. GSHsAg and WHsAg major polypeptides share $55 \%$ of their peptides with each other, but only $25 \%$ with HBsAg. This implies that the shared determinant(s), possibly $a$, is in $25 \%$ or less of the primary protein sequences of the major polypeptide.

If the extent of structural differences among the polypeptides of these viruses is a measure of the evolutionary change from a common ancestor, then the divergence of $d$ and $y$ subtype determinants of $\mathrm{HBsAg}$ occurred at approximately the same time as the divergence of HBsAg, GSHsAg, and WHsAg (Table 3; Fig. 5). That approximately $60 \%$ of the spots on the peptide maps of $a d w$ and $a d r$ are also found in ayw polypeptides, whereas only $30 \%$ of the spots on the ayw peptide maps are shared with $a d w$ and $a d r$, is a reflection of the greater number of spots consistently seen on ayw than on $a d w$ or $a d r$ peptide maps. The number of particle populations which show distinct isoelectric points (3) as well as the documented polymorphism $(a 1, a 2, a 3)$ of the $a$ determinant (17) in $\mathrm{HBsAg}$ from a single source are both greater for the $a y$ than for the ad subtype, suggesting that at least some of the increased number of spots consistently seen in the ayw maps could be the result of these documented heterogeneities. Alternatively, the $y$ determinant may be much more antigenically complex than the $d$ determinant. Recombination among HBVs and immunological selection of HBsAg determinants may have contributed to the increasing heterogeneity among surface antigen particles and the evolution of antigenic complexity. The presence of multiple or complex subtypes in single patients seems to suggest that their generation may arise from genetic recombination during infection of hosts with more than one virus (15). The finding of DNA sequence heterogeneity within different isolates of single subtypes (19) predicts at least some amino acid sequence heterogeneity within a given subtype, and the results of peptide mapping here are consistent with this prediction (Table 2). The close relationship seen among GSHsAg or WHsAg isolates parallels that noted within human subtypes or between $a d w$ and $a d r$ (Tables 1 and 2).

The differences observed in the peptide maps of HBsAg, GSHsAg, and WHsAg polypeptides (see Fig. 3C and 4D) are reflected not only in the number and position of spots conserved among HBsAg subtypes or among surface antigen polypeptides of the different viruses, but also in the charge of the peptides conserved in each case. Although GSHsAg and WHsAg share a larger percentage of charged polypeptides (Fig. 3C) than HBsAg subtypes (Fig. 4D), the composite showing peptides common to all surface antigen polypeptides studied here (Fig. 3D) indicates that approximately $44 \%$ (four of nine spots) are neutral and that the remainder probably have a single positive charge. The primary sequence of the major HBsAg polypeptide, deduced from nucleic acid and amino acid sequence analyses, defines major hydrophobic regions of the molecule at the $\mathrm{N}$-terminus, $\mathrm{C}$-terminus, and a region of 19 residues between positions 80 and 98 (4). Further, DNA sequence analyses of the HBsAg gene of various subtypes demonstrate conservation at the $\mathrm{N}$ - and $\mathrm{C}$-terminal regions of the HBsAg polypeptide, whereas more than $50 \%$ of the base pair changes resulting in amino acid substitutions occur in the middle region of the gene (15). Since the conditions used here for separating tryptic peptides (by charge and partition) would reflect any sequence heterogeneity, it is possible that the structural homology noted among all of the surface antigen particles (Fig. 3D) may derive from the amino- or carboxy-terminal end of the molecule (or from both), where the most highly conserved polypeptide sequences are found. The common or $a$ determinant, expected to be in a region of the HBsAg polypeptide highly conserved among subtypes, would probably be located in one or both of these regions as well. The data here, however, do not exclude other possibilities. Studies are now under way to learn the position of these conserved peptides in the major HBsAg polypeptide and to evaluate their antigenicity with a variety of antibody probes.

\section{ACKNOWLEDGMENTS}

We thank Susan Knight for excellent technical assistance and Walter Krengel III for production of monoclonal antibodies to GSHsAg.

This study was supported by Public Health Service research grant AI 13526 from the National Institutes of Health. M.A.F. was supported by an individual postdoctoral fellowship (grant PF-1792) from the American Cancer Society.

\section{LTTERATURE CITED}

1. Bancroft, W. H., F. K. Mundon, and P. K. Russell. 1972. Detection of additional antigenic determinants of hepatitis B antigen by immunoprecipitation. J. Immunol. 109:842-848.

2. Bolton, A. E., and W. M. Hunter. 1973. The labelling of proteins to high specific radioactivities by conjugation to a ${ }^{125}$ I-containing acylating agent. Biochem. J. 133: 529-539. 
3. Chairez, R., F. B. Hollinger, J. P. Bruschwig, and G. R. Dreesman. 1975. Comparative biophysical studies of hepatitis B antigen, subtypes $a d w$ and $a y w$. J. Virol. 15:182-190.

4. Charnay, P., E. Mandart, A. Hampe, F. Fitoussi, P. Tiollais, and F. Galibert. 1979. Location on the viral genome and nucleotide sequence of the gene coding for the two major polypeptides of the hepatitis $B$ surface antigen (HBsAg). Nucleic Acids Res. 7:335-346.

5. Couroucé-Pauty, A. M., P. V. Holland, J. B. Muller, and J. P. Soulier. 1976. International workshop on HBs antigen subtypes. Bibl. Haematol. (Basel) 42:76136.

6. Cummings, I. W., J. K. Browne, W. A. Salser, G. V. Tyler, R. L. Snyder, J. M. Smolec, and J. Summers. 1980. Isolation, characterization and comparison of recombinant DNAs derived from genomes of human hepatitis $B$ virus and woodchuck hepatitis virus. Proc. Natl. Acad. Sci. U.S.A. 77:1842-1846.

7. Fraker, P. J., and J. C. Speck. 1978. Protein and cell membrane iodinations with a sparingly soluble chloramide, 1,3,4,6-tetrachloro-3a,6a-diphenylglycoluril. Biochem. Biophys. Res. Commun. 80:849-857.

8. Gerlich, W. H., M. A. Feitelson, P. L. Marion, and W. S. Robinson. 1980. Structural relationships between the surface antigens of ground squirrel hepatitis virus and human hepatitis B virus. J. Virol. 36:787-795.

9. Gerlich, W., and R. Thomssen. 1975. Standardized detection of hepatitis B surface antigen: determination of its serum concentration in weight units per volume. Dev. Biol. Stand. 30:78-86.

10. Kaplan, P. M., R. L. Greenman, J. L. Gerin, R. H. Purcell, and W. S. Robinson. 1973. DNA polymerase associated with the human hepatitis B antigen. J. Virol. 12:995-1005.

11. Laemmli, U. K. 1970. Cleavage of structural proteins during the assembly of the head of bacteriophage $T_{4}$. Nature (London) 227:680-685.

12. Le Bouvier, G. L. 1971. The heterogeneity of Australia antigen. J. Infect. Dis. 123:671-675.

13. Marion, P. L., L. S. Oshiro, D. C. Regnery, G. H. Scullard, and W. S. Robinson. 1980. A virus of Beechey ground squirrels that is related to hepatitis B virus of humans. Proc. Natl. Acad. Sci. U.S.A. 77:29412945.

14. Means, G. E., and R. E. Feeney. 1968. Reductive alkylation of amino groups in proteins. Biochemistry 7:21922201.

15. Pasek, M., T. Goto, W. Gilbert, B. Zink, H. Schaller, P. Mackay, G. Leadbetter, and K. Murray. 1979. Hepatitis B virus genes and their expression in E. coli. Nature (London) 282:575-579.

16. Robinson, W. S. 1977. The genome of hepatitis $B$ virus. Annu. Rev. Microbiol. 31:357-377.

17. Soulier, J. P., and A. M. Couroucé-Pauty. 1973. New determinants of hepatitis B antigen (Au or $\mathrm{HB}$ antigen). Vox Sang. 25:212-234.

18. Shih, J. W., and J. L. Gerin. 1977. Proteins of hepatitis B surface antigen. J. Virol. 21:347-357.

19. Siddiqui, A., P. L. Marion, and W. S. Robinson. 1981. Ground squirrel hepatitis virus DNA: molecular cloning and comparison with hepatitis B virus DNA. J. Virol. 38:393-397.

20. Summers, J., J. M. Smolec, and R. Snyder. 1978. A virus similar to human hepatitis B virus associated with hepatitis and hepatoma in woodchucks. Proc. Natl. Acad. Sci. U.S.A. 75:4533-4537.

21. Werner, B. G., J. M. Smolec, R. Snyder, and J. Summers. 1979. Serological relationships of woodchuck hepatitis virus to human hepatitis B virus. J. Virol. 32: 314-322. 